\title{
Regulação dos serviços de radioterapia e quimioterapia pelas operadoras de planos de saúde no Brasil
}

\author{
Regulation of radiotherapy and chemotherapy services \\ by health plan organizations in Brazil
}

Sheyla Maria Lemos Lima ${ }^{1}$

Margareth Crisóstomo Portela ${ }^{1}$

Maria Alicia Domíngues Ugá ${ }^{1}$

Maurício Teixeira Leite de Vasconcellos ${ }^{2}$

${ }^{1}$ Departamento de

Administração e

Planejamento em Saúde.

Escola Nacional de Saúde

Pública. Fundação Oswaldo

Cruz (Fiocruz). R. Leopoldo

Bulhões 1480/718,

Manguinhos. 21.041-210

Rio de Janeiro RJ Brasil.

slemos@ensp.fiocruz.br

${ }^{2}$ Escola Nacional de

Ciências Estatísticas.

Fundação Instituto

Brasileiro de Geografia e

Estatística (IBGE).
Abstract This paper characterizes regulatory procedures applied by private health plan operators on their outpatient radiotherapy and chemotherapy services, especially via contracts, and outlines the health care providers' perception on regulation. The study relied on primary data, taking into consideration 638 hospitals and outpatient health care units with the services in question. A stratified random sample was selected, resulting in the inclusion of 54 units that are representative of the population, excluding hospitals that only provide radiotherapy. Private chemotherapy services are largely funded by health insurance plans $(75.0 \%)$, while radiotherapy services are predominantly covered by the public health system (49.0\%). Contracts are not applied by third part payers, in their potential, as regulatory and health care coordination instruments. The mechanisms of regulation applied by third part payers are centered on services use control and administrative aspects. It is recognized the need of adjustments for a health care quality focus, and contracts may contribute in this sense.

Key words Radiotherapy, Chemotherapy, Regulation, Contracts, Health plan operators
Resumo Este artigo caracteriza a regulação das operadoras de planos de saúde sobre os seus prestadores ambulatoriais de radioterapia e quimioterapia, em especial a contratualização, e fornece a percepção dos prestadores em relação à regulação. O estudo baseou-se em dados primários, considerando 638 unidades de saúde hospitalares e ambulatoriais com os referidos serviços. Uma amostra aleatória estratificada foi selecionada, resultando na inclusão de 54 unidades representativas da população, excluindo hospitais somente com radioterapia. Serviços privados de quimioterapia são grandemente financiados por planos de saúde (75,0\% da receita em média), e os de radioterapia, pelo sistema público de saúde (49,0\% da receita em média). A contratualização não é utilizada em sua potencialidade como instrumento de regulação e coordenação do cuidado pelas operadoras. Os mecanismos de regulação estão centrados no controle da utilização e em aspectos administrativos. Reconhece-se a necessidade de focar mais a qualidade assistencial, e a contratualização pode contribuir neste sentido.

Palavras-chave Radioterapia, Quimioterapia, Regulação, Contratualização, Operadoras de planos de saúde 


\section{Introdução}

O segmento de planos privados de assistência à saúde no Brasil constituiu-se sob forte indução do Estado, mas em um contexto de baixa regulação'. A partir da Lei 9.656, de 1998, e da criação da Agência Nacional de Saúde Suplementar (ANS), em 2000, estabeleceu-se um ambiente de maior normatização, controle e fiscalização para a criação e funcionamento das operadoras de planos de saúde. A ANS assumiu o papel de regular as relações entre usuários e operadoras, tendo impacto, ainda que indiretamente, sobre os prestadores de serviços ${ }^{2}$.

Regulação é aqui compreendida como o modo que o Estado, através de suas instituições organizações, regras, leis e incentivos - intervém sobre um setor da economia, controlando ou direcionando suas atividades. É indiscutivelmente necessária no setor saúde, considerando que a saúde não é uma mercadoria qualquer a ser negociada no mercado, constituindo-se em bem de mérito, vital para a preservação do homem, e em direito do cidadão, formalizado na Constituição brasileira. Ao Estado cabe assegurar que a prestação de serviços de saúde pelo setor privado atenda $a$ uma função social, orientada pelo nexo do interesse público ${ }^{3}$. Além disso, a intervenção do Estado se impõe por características do mercado de saúde: ocorrência de riscos e incertezas, assimetria de informações e tendência à concentração em um número pequeno de grandes empresas ${ }^{4}$. Considerando a natureza do bem e estas características, $\mathrm{o}$ Estado deve atuar para assegurar que os interesses coletivos (população) sejam privilegiados em relação aos individuais (indivíduos, grupos, organizações), atendendo, em última instância, as necessidades de saúde da população ${ }^{1,4,5}$.

As relações de conflitos de interesse e de poder entre os diferentes atores da saúde suplementar - usuários, operadoras e prestadores - podem ser analisadas a partir da Teoria da Agência, onde um ator (o titular) tem poder sobre o comportamento de outro ator (o agente), mas ao mesmo tempo é dele dependente para alcançar seus propósitos, em um contexto de grande incerteza gerada pela assimetria de informações entre eles. Um ator pode assumir ao mesmo tempo papel de titular e de agente. Para minimizar a assimetria de informações e os comportamentos oportunistas dela decorrentes, os contratos são utilizados com vistas à formalização dos compromissos, ao incremento da prestação de contas, transparência, coordenação e regulação ${ }^{2}$.

No mundo inteiro, no setor saúde, público ou privado, os contratos vêm sendo cada vez mais utilizados, com o propósito de melhorar o desempenho dos sistemas e serviços de saúde e incrementar a responsabilização. $O$ contrato possibilita associar a execução/prestação de serviços ao planejamento das ações de saúde (com base nas necessidades de saúde e oferta da rede), e condicionar o pagamento dos serviços prestados à sua prestação de contas e avaliação, a partir de metas e indicadores quantitativos e qualitativos pré-estabelecidos, entre contratado e contratante. No Brasil o uso de contratos, no âmbito do setor público e privado, é denominado de contratualização ${ }^{6}$.

A contratualização é sustentada e estruturada por um tripé incluindo um plano de atividades (o que o contratante quer da contratada e o que ela se propõe a oferecer em termos de serviços, com os recursos financeiros previstos), um sistema de monitoramento e avaliação, para assegurar que as atividades sejam desenvolvidas tal como compromissadas, e um sistema de incentivos, condicionando o repasse de recursos ao desempenho alcançado pelo contratado ${ }^{6}$.

Em consonância com este movimento, a ANS tornou obrigatório o uso de contratos entre operadoras e prestadores, em uma iniciativa, dentre outras, voltada para a maior estruturação e regulação do setor, bem como para a melhoria dos serviços ofertados aos seus beneficiários.

Pressupõe-se, aqui, que às operadoras de planos de saúde caberia a gestão de redes de serviços de saúde (próprios ou contratados) para atender necessidades de saúde de populações adscritas (beneficiários), devendo, portanto, estarem aptas a exercer regulação, planejamento, coordenação e avaliação dos seus prestadores, com vistas a um bom desempenho assistencial. Neste sentido o uso da contratualização seria um dos instrumentos de gestão para as operadoras.

Este artigo visa a caracterizar as relações de regulação, principalmente através da contratualização, das operadoras em relação a seus prestadores de serviços ambulatoriais de alta complexidade na atenção oncológica, especificamente, a radioterapia e a quimioterapia, terapias de alto custo utilizadas no tratamento do paciente oncológico, de forma isolada ou associada a outras intervenções.

O foco na atenção oncológica justifica-se pela importância epidemiológica do câncer no país, bem como complexidade e custos associados ao seu tratamento ${ }^{7}$. Além disso, em diagnóstico prévio da ANS, foi identificado grande contingente de autorizações de procedimentos de alta complexidade em oncologia no SUS ${ }^{7}$ para indivíduos com planos de saúde. 


\section{Métodos}

A pesquisa constituiu-se em um estudo transversal, desenvolvido através de um inquérito de abrangência nacional.

A população da pesquisa foi o conjunto de serviços de quimioterapia, radioterapia ou ambos, inseridos ou não em hospitais, extraída do cadastro da ANS de abril de 2008, onde constavam 648 estabelecimentos.

Foi construída uma amostra estratificada, pela combinação de tipo de serviço (quimio ou radio) e tipo de unidade (hospitais e outros). Para assegurar o espalhamento geográfico da amostra, ordenou-se o cadastro por estrato e código do município. Tal ordenação, combinada com a seleção equiprovável e sistemática das unidades de saúde, correspondeu a uma estratificação implícita da amostra por macrorregião.

O tamanho da amostra foi fixado em 70 unidades de saúde em decorrência das restrições orçamentárias da pesquisa. A alocação desse tamanho de amostra por estrato foi proporcional ao número de unidades de cada estrato no cadastro de seleção, resultando, após os arredondamentos, em uma amostra de 74 unidades de saúde, representativa dos 648 establecimentos da população.

$\mathrm{Na}$ realização do trabalho de campo houve muitas recusas, além de erros de cadastro, determinando que 54 dos 74 estabelecimentos originalmente previstos fossem entrevistados. Em função disso, a amostra realizada perdeu a possibilidade de inferir sobre os 10 hospitais somente com serviços de radioterapia do cadastro, reduzindo a população da pesquisa para 638 estabelecimentos. As demais perdas distribuíram-se entre os estratos não comprometendo a representatividade da amostra.

A coleta de dados ocorreu entre agosto de 2008 e outubro de 2009, por meio de entrevista com os dirigentes dos estabelecimentos/serviços.

O questionário utilizado contemplou a seguinte caracterização: (i) aspectos gerais - tipo de serviço (quimioterapia ou radioterapia, unidade ambulatorial ou hospitalar), fontes de receita e vínculos com operadoras de planos de saúde e com o Sistema Único de Saúde (SUS); (ii) estrutura da assistência - outros serviços utilizados, equipamentos, número e tipo de profissional; conformidade com normatização; (iii) estruturas e mecanismos de gestão focados no monitoramento e melhoria da qualidade assistencial; (iv) aspectos da contratualização - dimensões consideradas, mecanismos de acompanhamento, incentivos; e (v) satisfação dos prestadores em relação à regulação praticada pelas operadoras.
Foram obtidas estatísticas descritivas dos dados, processados através do programa SAS ${ }^{\circledast}$, utilizando-se os pesos amostrais calculados pelo inverso das probabilidades de inclusão na amostra.

\section{Resultados}

Conforme já apontado, a amostra realizada permite inferências sobre 638 estabelecimentos da população original, visto que 10 unidades hospitalares somente com radioterapia não foram representadas. Os 638 estabelecimentos comportam 801 serviços, sendo 575 de quimioterapia e 226 de radioterapia, que atuam predominantemente de forma independente e, muitas vezes, com CNPJ distintos.

Os resultados aqui apresentados dizem respeito aos estabelecimentos em si, enquanto outros se referem aos serviços de quimioterapia e de radioterapia mais especificamente.

Os 638 estabelecimentos estão concentrados nas regiões Sudeste (57,0\%) e Sul (18,5\%), seguidas pelo Nordeste (16,8\%), Centro-Oeste (5,9\%) e Norte (1,8\%). Acompanham, aproximadamente, a distribuição das operadoras de planos de saúde no país, sendo ainda mais concentrada no Sudeste do que a dos prestadores hospitalares da Saúde Suplementar ${ }^{8}$.

Considerando o total de estabelecimentos (638), 43,5\% constituem-se em unidades ambulatoriais com quimioterapia, 21,1\% em unidades hospitalares com quimioterapia, 17,8\% em unidades hospitalares com quimioterapia e radioterapia, 9,9\% em unidades ambulatoriais de radioterapia e $7,7 \%$ em unidades ambulatoriais com quimioterapia e radioterapia.

As operadoras mais importantes na cobertura de serviços ambulatoriais oncológicos para estes estabelecimentos no país são UNIMED (78,0\%), CASSI (39,5\%), Bradesco (35,7\%) Sulamérica $(30,8 \%)$, GEAP $(24,2 \%)$ e Petrobrás $(20,7 \%)$.

Tanto para os serviços de quimioterapia quanto para os de radioterapia há uma forte concentração da receita em poucas operadoras, destacando-se que, em ambos os casos, quase 50,0\% das receitas provenientes de planos de saúde referem-se a uma única operadora.

No que concerne à receita total, os serviços de quimioterapia têm como principal fonte as operadoras de planos de saúde $(75,6 \%)$, seguidas pelo SUS $(19,8 \%)$ e pagamento direto do paciente (4,7\%). Diferentemente, nos serviços de radioterapia, o SUS constui-se na maior fonte de receita $(48,8 \%)$, com as operadoras sendo responsáveis por $42,2 \%$, e o pagamento direto por $8,5 \%$. 
No que se refere à prestação de serviços simultaneamente para as operadoras e para o SUS há uma importante diferença entre os serviços de quimioterapia (575), que minoritariamente (27,6\%) são também prestadores do SUS, e os de radioterapia (226), que majoritariamente (68,3\%) o são. Tal diferença é ratificada na disponibilizaçao de vagas para a central de regulaçao do SUS, feita somente por $21,7 \%$ dos serviços de quimioterapia, em contraposição a 51,8\% dos serviços de radioterapia. As empresas de quimioterapia e radioterapia que prestam serviços a planos de saúde e ao SUS, no entanto, diferem-se pouco ou não se diferem em relação à prática de agendamento único ( $52,0 \%$ vs $49,4 \%$ ), à utilizaçao de fila única de espera $(59,0 \%$ vs $41,0 \%)$ e uso das mesmas instalaçoes ( $83,6 \%$ vs $83,1 \%)$, ofertando tratamento similar aos pacientes do SUS e de planos de saúde, pelo menos nesses aspectos.

\section{Mecanismos de regulação junto aos serviços de quimioterapia e radioterapia}

Entre os serviços de quimioterapia e os de radioterapia, $91,5 \%$ e $82,7 \%$, respectivamente, referem que ao menos uma das principais operadoras, em termos de volume de atendimento e/ou faturamento, exige autorização para realização de procedimentos durante o tratamento do paciente. Adicionalmente, cerca de metade dos dois grupos pratica autorregulação, mencionando possuir instância interna para autorização de procedimentos durante a realização do tratamento do paciente. No que concerne à regulação pelo SUS, $65,5 \%$ dos serviços de quimioterapia e 72,6\% dos de radioterapia referem sofrer vistoria das SES e/ou SMS de forma regular.

A autorização de procedimentos pelas operadoras demora em média, segundo os serviços de quimioterapia e radioterapia, 3,5 e 5 dias, respectivamente, podendo variar entre 1 e 24 dias, no primeiro caso, e 2 e 23 dias no segundo, comprometendo a qualidade do cuidado, na sua dimensão de prontidão às necessidades dos pacientes.

Por outro lado, registra-se a solicitação de autorização do paciente para o tratamento, em termo de informe consentido, por $57,8 \%$ dos serviços de quimioterapia e $72,7 \%$ dos de radioterapia, sublinhando-se que, ainda que tal procedimento não corresponda necessariamente a um esclarecimento e compartilhamento real do cuidado com os pacientes, ele é elementar, considerando-se os riscos e as incertezas envolvidos no tratamento do câncer.
As Tabelas 1 e 2 apresentam como se distribuem as operadoras de planos de saúde em relação ao exercício de regulação assistencial junto aos prestadores de quimioterapia e radioterapia, focalizando aspectos relevantes para o monitoramento do processo de cuidado aos seus beneficiários. Observa-se que grande parte dos serviços (quimioterapia, $69,50 \%$; radioterapia, $78,4 \%$ ) declara que nenhuma operadora solicita a taxa de eventos adversos decorrentes do tratamento, o que é significativo. Cerca de metade dos serviços de quimioterapia e menos da metade dos de radioterapia declaram a exigência do exame histológico e estadiamento da doença dos pacientes, por todas as operadoras a eles relacionadas. O protocolo de estadiamento é ainda menos requerido pelas operadoras, com somente $24,3 \%$ e $11,5 \%$ dos serviços de quimioterapia e de radioterapia indicando que $100 \%$ das operadoras o solicitam, e $32,8 \%$ e $38,1 \%$ que nenhuma operadora o solicita. Por fim, o protocolo de tratamento dos pacientes é referido como demandado por $100 \%$ das operadoras apenas por $32,5 \% \mathrm{e}$ $17,3 \%$ dos serviços de quimioterapia e radioterapia, valendo sublinhar que $16,9 \%$ e $27,4 \%$ respectivamente referem que nenhuma operadora solicita tal protocolo.

Apesar da baixa regulação assistencial pelas operadoras, $76,4 \%$ dos prestadores de quimioterapia informam realizar controles repetidos da prescrição medicamentosa por diferentes profissionais, e $81,5 \%$ monitorar eventos adversos apresentados pelos pacientes.

A baixa preocupação das operadoras de planos de saúde com as práticas assistenciais dos seus serviços de quimioterapia e radioterapia também se expressa nas suas exigências contratuais, apresentadas a seguir.

\section{Caracterização da contratualização entre as operadoras e os estabelecimentos com serviços de quimioterapia e/ou radioterapia}

A contratualização é uma pactuação entre contratante e contratado, expressa em um documento que identifica as partes contratantes, finalidades, exigências de documentação, instalações físicas e equipamentos, número e qualificação profissional; a definição dos serviços contratados com metas e indicadores quantitativos e qualitativos; os recursos envolvidos, critérios para o repasse associados à avaliação, periodicidade, reajustes; os incentivos; o sistema de monitoramento e avaliação - instância, periodicidade, critérios, etc; os compromissos das partes; as pos- 
Tabela 1. Distribuição dos serviços de quimioterapia atuantes na Saúde Suplementar $(\mathrm{N}=575)$ segundo percentual de operadoras e condições por elas exigidas. Brasil, 2009.

\begin{tabular}{|c|c|c|c|c|c|c|c|c|c|c|c|c|c|c|}
\hline \multirow{3}{*}{ Condiçõoes exigidas } & \multicolumn{14}{|c|}{ Percentual de operadoras } \\
\hline & \multicolumn{2}{|c|}{$0 \%$} & \multicolumn{2}{|c|}{$1-25 \%$} & \multicolumn{2}{|c|}{$26-50 \%$} & \multicolumn{2}{|c|}{$51-75 \%$} & \multicolumn{2}{|c|}{$76-99 \%$} & \multicolumn{2}{|c|}{$100 \%$} & \multicolumn{2}{|c|}{ NSI } \\
\hline & $\mathbf{N}$ & $\%$ & $\mathbf{N}$ & $\%$ & $\mathbf{N}$ & $\%$ & $\mathbf{N}$ & $\%$ & $\mathbf{N}$ & $\%$ & $\mathbf{N}$ & $\%$ & $\mathbf{N}$ & $\%$ \\
\hline Taxa de eventos adversos & 399 & 69,5 & 118 & 20,6 & 0 & 0 & 12 & 2,0 & 12 & 2,0 & 22 & 3,9 & 12 & 2,0 \\
\hline $\begin{array}{l}\text { Autorização para o tratamento de } \\
\text { eventos adversos }\end{array}$ & 120 & 21,0 & 59 & 10,2 & 0 & 0 & 0 & 0 & 34 & 5,9 & 316 & 55,0 & 46 & 7,9 \\
\hline $\begin{array}{l}\text { Exame histológico dos pacientes } \\
\text { atendidos }\end{array}$ & 49 & 8,5 & 23 & 3,9 & 0 & 0 & 60 & 10,5 & 104 & 18,0 & 303 & 52,8 & 36 & 6,3 \\
\hline $\begin{array}{l}\text { Estadiamento dos pacientes } \\
\text { atendidos }\end{array}$ & 60 & 10,4 & 11 & 2,0 & 11 & 2,0 & 82 & 14,2 & 81 & 14,0 & 306 & 53,2 & 24 & 4,2 \\
\hline $\begin{array}{l}\text { Protocolo de estadiamento dos } \\
\text { pacientes atendidos }\end{array}$ & 188 & 32,8 & 94 & 16,3 & 23 & 3,9 & 34 & 5,9 & 49 & 8,5 & 140 & 24,3 & 47 & 8,3 \\
\hline $\begin{array}{l}\text { Protocolo do tratamento dos } \\
\text { pacientes atendidos }\end{array}$ & 97 & 16,9 & 79 & 13,8 & 24 & 4,2 & 45 & 7,9 & 95 & 16,5 & 187 & 32,5 & 48 & 8,2 \\
\hline
\end{tabular}

Tabela 2. Distribuição dos serviços de radioterapia atuantes na Saúde Suplementar $(\mathrm{N}=226)$ segundo percentual de operadoras e condições por elas exigidas. Brasil, 2009.

\begin{tabular}{|c|c|c|c|c|c|c|c|c|c|c|c|c|c|c|}
\hline \multirow{3}{*}{ Condições exigidas } & \multicolumn{14}{|c|}{ Percentual de operadoras } \\
\hline & \multicolumn{2}{|c|}{$0 \%$} & \multicolumn{2}{|c|}{$1-25 \%$} & \multicolumn{2}{|c|}{$26-50 \%$} & \multicolumn{2}{|c|}{$51-75 \%$} & \multicolumn{2}{|c|}{$76-99 \%$} & \multicolumn{2}{|c|}{$100 \%$} & \multicolumn{2}{|c|}{ NSI } \\
\hline & $\mathbf{N}$ & $\%$ & $\mathbf{N}$ & $\%$ & $\mathbf{N}$ & $\%$ & $\mathbf{N}$ & $\%$ & $\mathbf{N}$ & $\%$ & $\mathbf{N}$ & $\%$ & $\mathbf{N}$ & $\%$ \\
\hline Taxa de eventos adversos & 177 & 78,4 & 13 & 5,8 & 0 & 0,0 & 13 & 5,8 & 0 & 0,0 & 23 & 10,0 & 0 & 2,0 \\
\hline $\begin{array}{l}\text { Autorização para o tratamento de } \\
\text { eventos adversos }\end{array}$ & 76 & 33,9 & 13 & 5,8 & 0 & 0,0 & 0 & 0,0 & 0 & 0,0 & 123 & 54,6 & 13 & 7,9 \\
\hline $\begin{array}{l}\text { Exame histológico dos pacientes } \\
\text { atendidos }\end{array}$ & 36 & 15,8 & 26 & 11,5 & 0 & 0,0 & 39 & 17,3 & 36 & 15,8 & 76 & 33,9 & 13 & 6,3 \\
\hline $\begin{array}{l}\text { Estadiamento dos pacientes } \\
\text { atendidos }\end{array}$ & 36 & 15,8 & 13 & 5,8 & 13 & 5,8 & 13 & 5,8 & 36 & 15,8 & 102 & 45,4 & 13 & 4,2 \\
\hline $\begin{array}{l}\text { Protocolo de estadiamento dos } \\
\text { pacientes atendidos }\end{array}$ & 86 & 38,1 & 52 & 23,0 & 0 & 0,0 & 0 & 0,0 & 49 & 21,6 & 26 & 11,5 & 13 & 8,3 \\
\hline $\begin{array}{l}\text { Protocolo do tratamento dos } \\
\text { pacientes atendidos }\end{array}$ & 62 & 27,4 & 37 & 16,5 & 13 & 5,8 & 0 & 0,0 & 62 & 27,3 & 39 & 17,3 & 13 & 8,2 \\
\hline
\end{tabular}

sibilidades de ajustes; o tempo de duração do contrato; as condições de revisão, rescisão e renovação; penalidades; instância de resolução de conflitos.

A Tabela 3 contempla $615(96,4 \%)$ estabelecimentos que reportaram exigências de documentação pelas operadoras, indicando que mais de $75 \%$ informaram terem sido requeridos a apresentar todos os documentos listados, por todas as operadoras, à exceção da descrição das dependências.

Destaca-se que 1,9\% dos estabelecimentos informaram que nenhuma operadora solicita o registro da Vigilância Sanitária.
Somente $37,5 \%$ dos estabelecimentos citaram que o cadastro de todos os seus profissionais é solicitado por todas as operadoras, sendo significativo que $10,4 \%$ dos estabelecimentos informem que nenhuma operadora exige os cadastros de todos os profissionais.

Observa-se que os contratos são subutilizados na sua potencialidade pela maior parte das operadoras. São poucos aspectos que constam como exigências contratuais a serem cumpridas pelos prestadores. Aspectos citados como presentes nos contratos de todas operadoras por mais de $70 \%$ são (Tabela 4): critérios de rescisão (85,3\%); definição dos valores dos serviços contra- 
Tabela 3. Distribuição de estabelecimentos com serviço ambulatorial oncológico atuantes na Saúde Suplementar segundo tipo de documento exigido pelas operadoras para credenciamento e/ou contrato $(\mathrm{N}=615)$. Brasil, 2009.

\begin{tabular}{|c|c|c|c|c|c|c|c|c|c|c|}
\hline \multirow{2}{*}{ Tipo de documento } & \multicolumn{2}{|c|}{$\begin{array}{l}\text { Todas as } \\
\text { operadoras }\end{array}$} & \multicolumn{2}{|c|}{$\begin{array}{l}\text { A maioria das } \\
\text { operadoras }\end{array}$} & \multicolumn{2}{|c|}{$\begin{array}{l}\text { A minoria das } \\
\text { operadoras }\end{array}$} & \multicolumn{2}{|c|}{$\begin{array}{l}\text { Nenhuma } \\
\text { operadora }\end{array}$} & \multicolumn{2}{|c|}{ NSI } \\
\hline & $\mathbf{N}$ & $\%$ & $\mathbf{N}$ & $\%$ & $\mathbf{N}$ & $\%$ & $\mathbf{N}$ & $\%$ & $\mathbf{N}$ & $\%$ \\
\hline Contrato social ou ata de constituição & 589 & 95,8 & 26 & 4,2 & - & - & - & - & - & - \\
\hline Alteração do contrato social & 542 & 88,2 & 24 & 3,9 & 36 & 5,8 & 13 & 2,1 & - & - \\
\hline CNPJ & 602 & 97,9 & 13 & 2,1 & - & - & - & - & - & - \\
\hline ISS & 471 & 76,6 & 22 & 3,7 & 62 & 10,0 & 34 & 5,5 & 26 & 4,2 \\
\hline Inscrição do responsável técnico no CRM & 533 & 86,6 & 71 & 11,6 & 11 & 1,8 & - & - & - & - \\
\hline $\begin{array}{l}\text { Descrição das dependências do } \\
\text { estabelecimento }\end{array}$ & 248 & 40,3 & 151 & 24,6 & 178 & 29,0 & 38 & 6,1 & - & - \\
\hline Lista de especialidades e serviços prestados & 484 & 78,6 & 118 & 19,3 & - & - & - & - & 13 & 2,1 \\
\hline $\begin{array}{l}\text { Cadastro Nacional de Estabelecimentos de } \\
\text { Saúde (CNES) }\end{array}$ & 521 & 84,7 & 71 & 11,6 & 11 & 1,8 & - & - & 12 & 1,9 \\
\hline Registro na Vigilância Sanitária & 531 & 86,3 & 35 & 5,7 & 24 & 4,0 & 12 & 1,9 & 13 & 2,1 \\
\hline Alvará de funcionamento & 567 & 92,1 & 37 & 6,1 & 11 & 1,8 & - & - & - & - \\
\hline Outro & 107 & 17,4 & 83 & 13,4 & 107 & 17,4 & 213 & 34,6 & 105 & 17,2 \\
\hline Nenhum documento & - & - & - & - & & - & 488 & 79,4 & 127 & 20,6 \\
\hline
\end{tabular}

tados $(85,2 \%)$; serviços contratados $(83,7 \%)$; procedimentos contratados $(78,1 \%)$; responsabilidades das partes contratantes (77,6\%); unidade de pagamento (75,3\%); e momento do pagamento (74,3\%). A definição dos prazos e procedimentos para pagamento foi referida por $69,9 \%$ dos estabelecimentos.

Chama a atenção que aspectos relacionados à assistência tais como critérios de indicação de procedimentos/uso de diretrizes clínicas e sistema de referenciamento de paciente, são referidos como solicitados por $100 \%$ das operadoras por apenas $7,4 \%$ e $12,6 \%$ dos estabelecimentos, respectivamente. Vale ainda ressaltar que praticamente não há repasse de recursos condicionado a qualquer avaliação, tendo em vista que critérios de ônus e bônus financeiros, condicionamento do repasse de recursos a indicadores quantitativos de produção assistencial e a indicadores qualitativos da assistência são referidos por 9,2\%, 7,4\% e $7,1 \%$ dos estabelecimentos, respectivamente, como presentes no contrato de $100 \%$ das operadoras (Tabela 4).

Outros aspectos pertinentes à contratualização entre estabelecimentos e operadoras são destacados a seguir: (1) 55,5\%, 31,8\% e 10,9\% dos estabelecimentos referem, respectivamente, que todas ou a maioria das operadoras, somente a minoria e nenhuma operadora realiza visita técnica antes da assinatura do primeiro contrato; (2) o pagamento retrospectivo é o predominante, com mais de 98,0\% dos estabelecimentos declarando que todas ou a maioria das operadoras que compram os seus serviços adotam tal modalidade, ainda que 5,9\% dos estabelecimentos refiram o pagamento prospectivo para uma minoria de operadoras; (3) não se observam como usuais diferenças de valores pagos ou de modalidade de pagamento entre planos de uma mesma operadora; e (4) cerca de metade dos estabelecimentos informa que todas as operadoras solicitam informação sobre o diagnóstico do paciente $(46,8 \%)$ e o quantitativo de procedimentos realizados $(51,7 \%)$, e somente $18,8 \%$ sobre a orientação médica dada ao paciente, chamando a atenção que $31,1 \%$ e 44,5\% dos estabelecimentos informem que nenhuma operadora solicita, respectivamente, a informação sobre o quantitativo dos procedimentos e sobre a orientação médica dada ao paciente.

Considerando-se o conjunto de prestadores de serviços de quimioterapia e/ou radioterapia observa-se que embora o percentual médio de glosa não seja elevado $(6,8 \%)$, a taxa de recuperação é próxima à metade do que é glosado $(54,5 \%)$, o que parece indicar não haver um critério claro para glosar ou aprovar. Chama a atenção que a média de pagamento seja 60,2 dias, o que é consideravelmente elevado. A maior parte dos estabelecimentos (54,9\%) informa que as operadoras praticam reajuste anual de preços.

Com relação a visitas periódicas para avaliação dos prestadores e realização de inspeções, observa-se o baixo uso de ambos os mecanismos por parte das operadoras de planos de saúde. No que diz respeito à realização de inspeções, 
Tabela 4. Distribuição de estabelecimentos com serviço ambulatorial oncológico atuantes na Saúde Suplementar segundo aspectos contemplados nos contratos com operadoras de planos de saúde $(\mathrm{N}=638)$. Brasil, 2009.

\begin{tabular}{|c|c|c|c|c|c|c|c|c|c|c|}
\hline \multirow{2}{*}{ Aspectos do contrato } & \multicolumn{2}{|c|}{$\begin{array}{c}\text { Todas as } \\
\text { operadoras }\end{array}$} & \multicolumn{2}{|c|}{$\begin{array}{l}\text { A maioria das } \\
\text { operadoras }\end{array}$} & \multicolumn{2}{|c|}{$\begin{array}{l}\text { A minoria das } \\
\text { operadoras }\end{array}$} & \multicolumn{2}{|c|}{$\begin{array}{l}\text { Nenhuma } \\
\text { operadora }\end{array}$} & \multicolumn{2}{|c|}{ NSI } \\
\hline & $\mathbf{N}$ & $\%$ & $\mathbf{N}$ & $\%$ & $\mathbf{N}$ & $\%$ & $\mathbf{N}$ & $\%$ & $\mathbf{N}$ & $\%$ \\
\hline Serviços a serem contratados & 534 & 83,7 & 82 & 12,7 & - & - & 11 & 1,8 & 11 & 1,8 \\
\hline Procedimentos a serem contratados & 498 & 78,1 & 81 & 12,8 & 13 & 2,0 & 34 & 5,3 & 11 & 1,8 \\
\hline Padrão das instalações físicas & 200 & 31,3 & 120 & 18,8 & 167 & 26,2 & 140 & 21,9 & 11 & 1,8 \\
\hline $\begin{array}{l}\text { Registro dos prestadores no Cadastro Nacional } \\
\text { de Estabelecimentos de Saúde }\end{array}$ & 437 & 68,5 & 81 & 12,7 & 64 & 9,9 & 45 & 7,1 & 11 & 1,8 \\
\hline Registro da operadora na ANS & 422 & 66,1 & 95 & 15,0 & 49 & 7,7 & - & - & 72 & 11,2 \\
\hline $\begin{array}{l}\text { Momento do pagamento (anterior ou } \\
\text { posterior ao serviço prestado) }\end{array}$ & 474 & 74,3 & 130 & 20,3 & - & - & - & - & 34 & 5,4 \\
\hline $\begin{array}{l}\text { Unidade de pagamento (por ato médico, per } \\
\text { capita, por caso tratado, por orçamento } \\
\text { global ou outro) }\end{array}$ & 481 & 75,3 & 135 & 21,1 & 11 & 1,8 & - & - & 11 & 1,8 \\
\hline Definição dos valores dos serviços contratados & 544 & 85,2 & 60 & 9,4 & 23 & 3,6 & - & - & 11 & 1,8 \\
\hline $\begin{array}{l}\text { Definição de prazos e procedimentos para } \\
\text { faturamento e pagamento }\end{array}$ & 446 & 69,9 & 146 & 22,9 & 35 & 5,4 & - & - & 11 & 1,8 \\
\hline Rotina para auditoria técnica e administrativa & 211 & 33,0 & 106 & 16,6 & 146 & 22,9 & 152 & 23,9 & 23 & 3,6 \\
\hline $\begin{array}{l}\text { Rotina para habilitação do beneficiário junto } \\
\text { ao hospital }\end{array}$ & 244 & 38,2 & 81 & 12,8 & 96 & 15,1 & 119 & 18,6 & 98 & 15,3 \\
\hline $\begin{array}{l}\text { Procedimentos que necessitam de autorização } \\
\text { administrativa }\end{array}$ & 331 & 51,8 & 141 & 22,2 & 85 & 13,3 & 70 & 10,9 & 11 & 1,8 \\
\hline $\begin{array}{l}\text { Indicadores quantitativos de produção } \\
\text { assistencial como condicionantes do repasse } \\
\text { de recursos }\end{array}$ & 47 & 7,4 & 47 & 7,4 & 47 & 7,4 & 474 & 74,2 & 23 & 3,6 \\
\hline $\begin{array}{l}\text { Indicadores qualitativos da assistência como } \\
\text { condicionantes do repasse de recursos }\end{array}$ & 45 & 7,1 & - & - & 98 & 15,3 & 472 & 74,0 & 23 & 3,6 \\
\hline $\begin{array}{l}\text { Critérios de indicação de procedimentos - uso } \\
\text { de diretrizes clínicas }\end{array}$ & 47 & 7,4 & 58 & 9,1 & 134 & 20,9 & 365 & 57,2 & 34 & 5,4 \\
\hline $\begin{array}{l}\text { Sistema de referenciamento de pacientes (a } \\
\text { uma rede de prestadores) }\end{array}$ & 81 & 12,6 & 23 & 3,6 & 86 & 13,5 & 414 & 64,9 & 34 & 5,4 \\
\hline Critérios de glosa & 134 & 21,1 & 68 & 10,6 & 98 & 15,3 & 291 & 45,6 & 47 & 7,4 \\
\hline Responsabilidades das partes contratantes & 495 & 77,6 & 119 & 18,6 & - & - & - & - & 24 & 3,8 \\
\hline $\begin{array}{l}\text { Rotinas para pleno atendimento ao disposto } \\
\text { no art. } 18 \text { da Lei } 9656 / 98\end{array}$ & 154 & 24,1 & 68 & 10,7 & 96 & 15,0 & 140 & 21,9 & 180 & 28,2 \\
\hline Penalidades por não cumprimento contratual & 411 & 64,3 & 154 & 24,2 & 26 & 4,1 & 11 & 1,8 & 36 & 5,6 \\
\hline $\begin{array}{l}\text { Regras para reajuste, contendo forma e } \\
\text { periodicidade }\end{array}$ & 177 & 27,7 & 129 & 20,2 & 118 & 18,5 & 167 & 26,2 & 47 & 7,4 \\
\hline $\begin{array}{l}\text { Não discriminação de pacientes e vedação da } \\
\text { exclusividade na relação contratual }\end{array}$ & 250 & 39,2 & 71 & 11,1 & 70 & 11,0 & 198 & 31,0 & 49 & 7,7 \\
\hline Prazo de vigência do contrato & 440 & 69,0 & 117 & 18,3 & 11 & 1,8 & 34 & 5,3 & 36 & 5,6 \\
\hline Critérios de rescisão do contrato & 45 & 85,3 & 60 & 9,3 & 11 & 1,8 & 11 & 1,8 & 11 & 1,8 \\
\hline Critérios de renovação do contrato & 429 & 67,2 & 58 & 9,1 & 47 & 7,4 & 81 & 12,7 & 23 & 3,6 \\
\hline $\begin{array}{l}\text { Critérios para bônus e ônus (incentivos e } \\
\text { penalidades) financeiros }\end{array}$ & 58 & 9,2 & 34 & 5,4 & 96 & 15,0 & 389 & 61,0 & 60 & 9,4 \\
\hline $\begin{array}{l}\text { Mecanismos e responsáveis pelo } \\
\text { acompanhamento dos contratos }\end{array}$ & 178 & 27,9 & 96 & 15,0 & 107 & 16,8 & 198 & 31,1 & 59 & 9,2 \\
\hline
\end{tabular}

mais de $80 \%$ dos estabelecimentos informaram que a minoria ou nenhuma operadora fez inspeção com vistas à avaliação de aspectos como acolhimento ao paciente, tempo de espera, direitos dos pacientes, equipamentos em uso, limpeza e conservação e conforto na hotelaria.

Enfim, a Tabela 5 apresenta os níveis de satisfação dos estabelecimentos em relação aos aspec- 
Tabela 5. Distribuição de estabelecimentos com serviço ambulatorial oncológico atuantes na Saúde Suplementar segundo a nota atribuída a aspectos do contrato com as operadoras $(\mathrm{N}=638)$. Brasil, 2009.

\begin{tabular}{lccc}
\hline \multicolumn{1}{c}{ Aspectos do contrato } & & & \\
& N & Nota média & $\begin{array}{c}\text { Intervalo de } \\
\text { confiança 95\% }\end{array}$ \\
\hline Momento de repasse dos recursos/ pagamento & 627 & 2,96 & $2,69-3,24$ \\
Unidade de medida para o repasse de recursos & 579 & 3,12 & $2,74-3,50$ \\
Valores financeiros repassados & 627 & 2,83 & $2,47-3,20$ \\
Acompanhamento da qualidade assistencial & 344 & 2,51 & $2,05-2.97$ \\
Critérios de indicação de procedimentos - uso de diretrizes clínicas & 368 & 2,77 & $2,38-3,17$ \\
Critérios de glosa & 464 & 2,26 & $1,87-2,65$ \\
Incentivos & 264 & 1,96 & $1,42-2,51$ \\
Responsabilidades das partes contratantes & 604 & 3,58 & $3,22-3,94$ \\
Prazo de vigência do contrato & 570 & 4,16 & $3,85-4.48$ \\
Critérios de rescisão do contrato & 504 & 3,89 & $3,54-4,25$ \\
Critérios de renovação do contrato & 547 & 4,05 & $3,70-4,40$ \\
Penalidades & 495 & 3,26 & $2,80-3,72$ \\
Mecanismos e responsáveis pelo acompanhamento dos contratos & 441 & 3,15 & $2,62-3.68$ \\
\hline
\end{tabular}

tos presentes nos contratos com operadoras de planos de saúde. Considerando a atribuição de notas entre 1 e 5 , a vigência e critérios de renovação do contrato são aspectos avaliados mais favoravelmente, em oposição à previsão de incentivos, critérios de glosa e acompanhamento da qualidade assistencial, com avaliação desfavorável.

\section{Discussão}

Ainda que a Lei 9.656 e a criação da ANS tenham sido importantes avanços no marco regulatório do país no âmbito da saúde suplementar, a regulação da atenção à saúde e de sua qualidade ainda está longe do desejado. A ANS cada vez mais tem investido no campo assistencial, induzindo as operadoras a interferirem nos seus prestadores, haja vista as resoluções de estipular tempo máximo de atendimento, de ampliar o rol obrigatório de procedimentos, recomendando inclusive o uso de diretrizes clínicas para definir os critérios de obrigatoriedade de cobertura de alguns procedimentos, e instituir o Programa de Monitoramento da Qualidade de Serviços da Rede Suplementar (QUALISS) ${ }^{8}$. Apesar das iniciativas da Agência, o estudo evidenciou que as operadoras não tinham como objeto de sua regulação, em relação aos prestadores de radioterapia e quimioterapia por elas contratados, a qualidade da atenção prestada.

A preocupação com a cobertura e a regulação sobre planos de saúde públicos e privados está na agenda internacional, registrando-se, por exemplo, o projeto em desenvolvimento em 10 países da Organização para Cooperação e Desenvolvimento Econômico (Organisation for Economic Co-operation and Development, OECD) que se centra em tais aspectos, com vistas à redução de barreiras e inequidades no acesso e uso de serviços de saúde de qualidade?.

A pouca preocupação das operadoras em relação aos serviços assistenciais de seus prestadores ambulatoriais oncológicos de alta complexidade é aparentemente ratificada ao se observar que é baixa a solicitação de comprovação de diagnóstico de câncer e de protocolo de tratamento dos seus beneficiários. O diagnóstico é realizado por meio do exame histológico do tumor e de um conjunto de exames que possibilita seu estadiamento indicando a agressividade e o quanto que a doença já se espalhou pelo organismo permitindo assim fazer um prognóstico, e fundamentalmente, definir o tratamento adequado. Além disso, o estadiamento do tumor também possibilita estudos de sobrevida dos pacientes, o que possibilitaria às operadoras avaliarem a qualidade do cuidado ofertado pelos prestadores. De igual forma, ter acesso ao protocolo de tratamento utilizado pelo prestador e compará-lo às evidências científicas, é a única forma das operadoras terem a garantia que seus pacientes estão sendo tratados adequadamente.

No campo da qualidade assistencial, a regulamentação do tempo de espera máximo para os procedimentos de alta complexidade (até 21 dias) possivelmente deverá desencadear o monitoramento deste aspecto pelas operadoras junto a 
sua rede de prestadores, revertendo o quadro observado no estudo onde mais de $70 \%$ dos estabelecimentos de quimioterapia e radioterapia informaram que as operadoras em suas inspeções não consideravam o tempo de espera como objeto de inspeção e avaliação. A implantação do QUALISS também poderá ser um fator indutor da melhoria da qualidade da assistência.

Outra questão a merecer atenção é a tendência consubstanciada após a regulação, de concentração de poucas operadoras líderes no mercado de planos de saúde. Destaca-se, a importância da UNIMED ${ }^{10}$, que mesmo não se constituindo em uma única empresa, expressa a abrangência e a capilaridade das cooperativas médicas no país, também no contexto da atenção oncológica. Há ainda nos serviços, uma forte concentração do faturamento em poucas operadoras e particularmente em uma delas. Se por um lado, têm-se operadoras mais sólidas do ponto de vista financeiro, com mais beneficiários, diluindo, assim, os riscos, o que, em tese, possibilitaria melhor cobertura e preços; por outro, a diminuição de concorrentes e a tendência ao oligopólio conferem mais poder às operadoras, reduzindo a capacidade de negociação do Estado, de prestadores e de beneficiários, em relação à qualidade dos serviços prestados, cobertura e preços praticados $^{3,5}$. Apesar de não haver consenso sobre os possíveis efeitos da concentração, esta é uma situação a ser considerada na regulação.

Não se pode também desprezar a relevância epidemiológica das doenças oncológicas no país, exigindo, para seu adequado enfrentamento, além de ações de promoção da saúde e de prevenção, intervenções voltadas para o tratamento, as quais requerem recursos de alta complexidade e custo, o que por si só já justificaria a necessidade de regulação. Por outro lado, tal necessidade faz-se mais imperiosa ainda ao se considerar o imbricamento existente entre Saúde Suplementar e SUS. Embora o ressarcimento obrigatório pelos planos de saúde ao SUS das internações na rede pública de seus pacientes estivesse previsto na Lei 9.656, só a partir do final de 2011, a ANS ampliou esta obrigatoriedade para os procedimentos ambulatoriais de alta complexidade, como é o caso da quimioterapia e radioterapia. Concomitantemente a esta medida, o Ministério da Saúde determinou, pela Lei 12.469, que os recursos arrecadados pela ANS com o ressarcimento fossem transferidos para o Fundo Nacional de Saúde (FNS), os quais deverão ser aplicados em ações estratégicas para o SUS. Tal medida faz com que, efetivamente, os valores cobrados pela ANS às operadoras de planos de saúde retornem ao SUS, o que poderá ser aplicado na atenção oncológica prestada pelo SUS.

Chama também à atenção a relativa autonomia, em relação ao Estado, dos serviços de quimioterapia quando comparados aos de radioterapia, no que se refere à fonte de receita. Os primeiros são grandemente financiados pelas operadoras de planos de saúde, sendo somente $28 \%$ deles prestadores de serviços aos SUS. Já os serviços de radioterapia, majoritariamente (68\%) também prestadores do SUS, têm aproximadamente metade da sua receita oriunda do SUS e a outra metade de planos de saúde ou pagamento direto do paciente. Os serviços de radioterapia (presentes em 35,4\% dos estabelecimentos com atenção ambulatorial oncológica) também despontam em muito menor quantidade do que os de quimioterapia (90,1\% dos estabelecimentos) o que pode ser explicado pelo elevado investimento financeiro que se faz necessário para a instalação dos equipamentos de radioterapia.

Apesar da obrigatoriedade instituída pela ANS da existência de contratos entre operadoras e seus prestadores, este estudo evidenciou que a lógica contratual não é explorada na sua potencialidade $^{6,11}$. A contratualização pode ser um importante dispositivo para associar a execução local ao planejamento, promovendo uma vinculação formal entre o que a operadora precisa que a sua rede faça e o que estes prestadores se comprometem a fazer, considerando as necessidades de saúde dos beneficiários da operadora. Possibilita ainda incrementar a transparência e a prestação de contas sobre resultados pré-definidos, entre operadoras, prestadores e beneficiários/ usuários. Entretanto, para seu uso potencial, exige que o contratante, a operadora, tenha um plano de atividades, utilize mecanismos de monitoramento e avaliação e institua um sistema de incentivos, associando o repasse de recursos ao desempenho dos prestadores ${ }^{6}$.

Nada disso ocorre na atual contratualização entre as operadoras e os prestadores. Há ainda considerável número de operadoras que nem mesmo requer informação sobre o quantitativo de procedimentos e sobre a orientação médica dada ao paciente. Até mesmo, segundo os próprios prestadores, é necessário um ajuste na lógica de contratualização, prevendo incentivos associados a resultados e incorporando, como metas a serem alcançadas, aspectos que possibilitem monitorar, mensurar e avaliar a qualidade assistencial na dimensão técnica e na satisfação dos beneficiários.

Além disso, o uso da lógica contratual pode propiciar uma melhor coordenação, por parte 
das operadoras, das ações desenvolvidas pela sua rede de prestadores ${ }^{11}$, assegurando a continuidade e a integralidade da atenção prestada, vinculando por meio de uma linha de cuidado, os diferentes pontos que compõem a atenção de uma dada condição e/ou doença.

A contratualização pode ser uma ferramenta que possibilite maior foco das operadoras e da ANS nos resultados qualitativos e quantitativos, financeiros e assistenciais dos prestadores de serviços no âmbito da Saúde Suplementar, induzindo operadoras e prestadores de serviços a desenvolverem competência técnica e a assumirem um maior compromisso com o bem público. Cada vez mais se impõe a regulação pública dos elementos a serem considerados nessa contratualização entre operadoras e prestadores, para que possa assegurar à população a qualificação da assistência.

Em um contexto de interesses tão diversos como o da Saúde Suplementar, alguns desafios precisam ser enfrentados, inclusive a ampliação da regulação para além dos planos novos e individuais, tendo em vista a predominância de planos coletivos $(74,9 \%)$ adquiridos por empresas 5 .

\section{Referências}

1. Costa NR. O Regime regulatório e o mercado de planos de saúde no Brasil. Cien Saude Colet 2008; 13(5):1453-1463.

2. Sato FRL. A Teoria da agência no setor da saúde: o caso do relacionamento da Agência Nacional de Saúde Suplementar com as operadoras de planos de assistência supletiva no Brasil. Rev Adm. Pública 2007; 41(1):49-62.

3. Ocké-Reis CO. Os desafios da ANS frente à concentração dos planos de saúde. Cien Saude Colet 2007; 12(4):1041-1050.

4. Santos FP, Merhy, EE. A regulação pública da saúde no Estado brasileiro - uma revisão. Interface (Botucatu) 2006; 10(19):25-41.

5. Pietrobon L, Prado ML, Caetano JC. Saúde Suplementar no Brasil: o papel da Agência Nacional der Saúde Suplementar. Physis 2008; 18(4):767-783.

6. Lima SML, Rivera FJU. A contratualização nos Hospitais de Ensino no Sistema Único de Saúde brasileiro. Cien Saude Colet 2011; 17(9):2507-2521.

7. Brasil. Ministério da Saúde (MS). Secretaria de Assistência à Saúde. Instituto Nacional do Câncer. Coordenação de Prevenção e Vigilância. Estimativa 2006: Incidência de Câncer no Brasil. Rio de Janeiro: INCA; 2005.
Afora tudo isso, há que se definir de modo mais claro uma atuação do Estado que contemple de forma integrada os segmentos público e privado no planejamento e na execução de suas políticas de saúde. Superar a fragmentação e a descontinuidade da atenção hoje existente é um desafio para o SUS e para a Saúde Suplementar.

\section{Colaboradores}

SML Lima, MC Portela, MAD Ugá e MTL Vasconcellos participaram igualmente de todas as etapas de realização deste artigo.

\section{Agradecimentos}

À leitura cuidadosa de Claudia Brito funcionária do Instituto Nacional de Câncer José Alencar Gomes da Silva do MS (INCA/MS).
8. Brasil. Agência Nacional de Saúde Suplementar. Resolução Normativa RN n ${ }^{\circ} 275$, de 1 de novembro 2011. Dispõe sobre a Instituição do Programa de Monitoramento da Qualidade dos Prestadores de Serviços na Saúde Suplementar - QUALISS. Diário Oficial da União 2011; 3 nov.

9. Quesnel-Vallée A, Renahy E, Jenkins T, Cerigo H. Assessing barriers to health insurance and threats to equity in comparative perspective: The Health Insurance Access Database. BMC Health Services Research 2012; 12:107.

10. Portela MC, Lima SML, Ugá AD, Gerschman S, Vasconcellos M. Estrutura e qualidade assistencial dos prestadores de serviços hospitalares. Cad Saude Publica 2010; 26(2):399-408.

11. Mendes EV. As redes de atenção à saúde. Belo Horizonte: ESP-MG; 2009.

Artigo apresentado em 28/06/2012

Aprovado em 27/08/2012

Versão final apresentada em 16/09/2012 